\title{
Uma Manifestação Atípica de Dermite de Contacto Alérgica a Metacrilatos numa Dentista
}

\author{
Susana Brás', Cátia Alves², Cristina Amaro ${ }^{3}$, Jorge Cardoso ${ }^{4}$ \\ 'Interna do Internato Complementar de Dermatovenereologia/Resident, Dermatology, Serviço de Dermatologia e Venereologia do \\ Hospital Curry Cabral - Centro Hospitalar de Lisboa Central, Portugal \\ 2Interna do Internato Complementar de Imunoalergologia/Resident, Immunoallergology Serviço de Imunoalergologia do Hospital \\ Dona Estefânia - Centro Hospitalar de Lisboa Central, Portugal \\ ${ }^{3}$ Assistente Hospitalar de Dermatologia e Venereologia/Consultant, Dermatology, Serviço de Dermatologia e Venereologia do \\ Hospital Curry Cabral - Centro Hospitalar de Lisboa Central, Portugal \\ ${ }^{4}$ Director de Serviço de Dermatologia e Venereologia/Head of Dermatology Department, Serviço de Dermatologia e Venereologia \\ do Hospital Curry Cabral - Centro Hospitalar de Lisboa Central, Portugal
}

RESUMO - A dermite de contacto alérgica a acrilatos e metacrilatos é frequente em profissionais de saúde como os dentistas e os protésicos dentários. Os autores apresentam o caso de uma dermite de contacto alérgica de localização atípica a metacrilatos, numa dentista, de modo a alertar para esta realidade e a implementar métodos para evitar a sensibilização a estes alergenos. PALAVRAS-CHAVE - Acrilatos; Dermite de Contacto Alérgica; Dermatite Ocupacional; Dentistas; Metacrilatos.

\section{Atypical Pattern of Methacrylate Allergic Contact Dermatitis in a Dentist}

ABSTRACT - Allergic contact dermatitis to acrylates and methacrylates is common in dentists and dental prosthetic professionals. The authors present a case of an atypical localization of allergic contact dermatitis to methacrylates in a dentist in order to alert to this reality and to implement methods to prevent sensitization to these allergens.

KEY-WORDS - Acrylates; Dermatitis, Allergic Contact; Dermatitis, Occupational; Dentists; Methacrylates.

\section{INTRODUÇÃO}

A dermite de contacto alérgica a acrilatos e metacrilatos é uma dermatose frequente em profissões como manicures, dentistas, protésicos dentários e trabalhadores da indústria gráfica, pela manipulação frequente de produtos com estes alergenos.' A prevalência da sensibilização a metacrilatos nos profissionais de medicina dentária varia entre os $5,8 \%$ e os $31,7 \%$. $^{2,3} \mathrm{Na}$ maioria dos casos as lesões localizam-se nas falanges distais ou nas pálpebras. ${ }^{1}$

\section{CASO CLÍNICO}

Os autores apresentam o caso de uma doente do sexo feminino, 30 anos, dentista há 5 anos, que recorre à consulta de dermatologia por dermatose com 24 horas de evolução localizada ao dorso da mão esquerda, na prega entre os $1^{\circ}$ e $2^{\circ}$ ossos metacárpicos. Tratava-se de uma placa circular eritematosa com prurido e que em poucas horas havia formado uma bolha central de conteúdo seroso (Fig. 1).

A doente referia uma lesão semelhante 3 semanas antes, tendo sido medicada com furoato de mometasona, $1 \mathrm{mg} / \mathrm{g}$, pomada com regressão total em 2 semanas.

Da história clínica, verificou-se que as lesões apareciam na sequência da atividade laboral, pelo que se suspeitou de dermite de contacto alérgica em contexto profissional.

A doente revelou que durante os procedimentos de restauração dentária colocava o produto Adper Single bond $2^{\circledR}$ da $3 \mathrm{M}$ no dorso da mão esquerda, sobre a luva (Fig. 2). Este produto continha na sua composição vários acrilatos: bisfenol A diglicil éter dimetacrilato (BISGMA), metacrilato de 2-hidroxietil (HEMA) e diuretano dimetacrilato (UDMA).

Foram realizadas provas epicutâneas com a série básica do grupo português das dermites de contacto e a série
Correspondência: Dr. ${ }^{a}$ Susana Brás

Serviço de Dermatologia e Venereologia - Centro Hospitalar de Lisboa Central

Alameda de Santo António dos Capuchos - 1169-050 Lisboa, Portugal

E-mail: bras.susana@gmail.com
Recebido/Received

Dezembro/December 2015

Aceite/Accepted

Janeiro/January 2016 


\section{GPDEC}

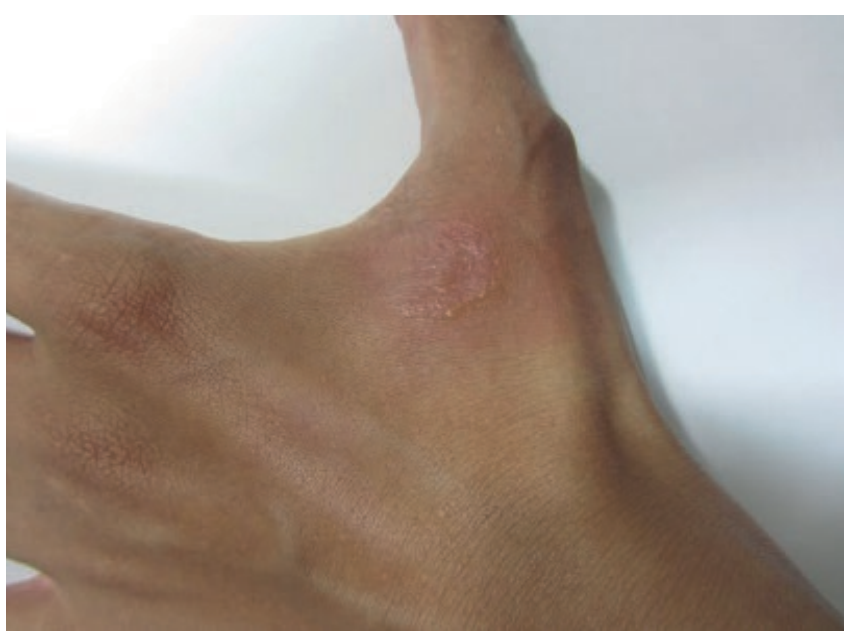

Figura 1 - Placa circular eritematosa centrada por bolha flácida de conteúdo seroso localizada no dorso da mão esquerda entre o primeiro e o segundo metacarpo

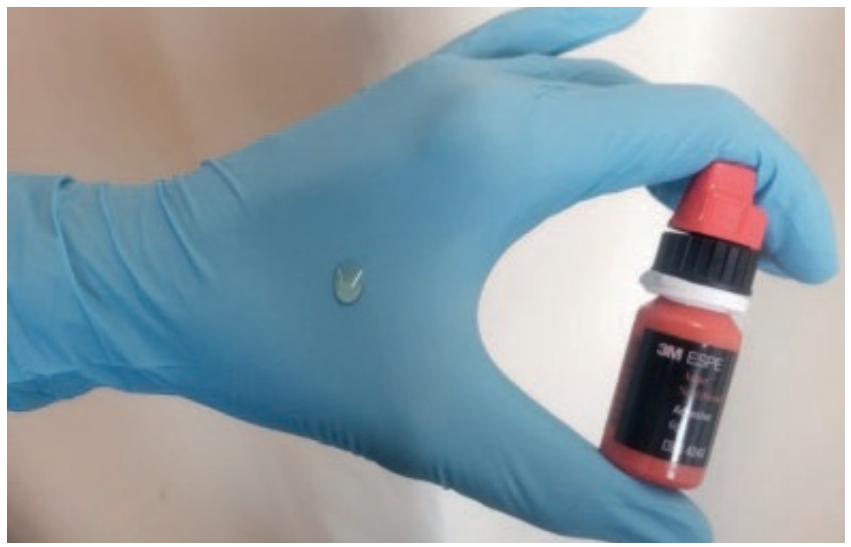

Figura 2 - Demonstração do modo de exposição ao alergeno durante a actividade profissional.

de produtos dentários (Chemotechnique, Suécia). As leituras foram realizadas às 48 e às 96 horas. Os resultados foram positivos $(2+)$ para: dimetacrilato de trietilenoglicol (TREGD$M A)$, dimetacrilato de etilenoglicol (EGDMA), HEMA e metacrilato de tetrahidrofurfuril (THFMA) (Fig. 3). O BISGMA e UDMA não foram testados no presente caso.

A doente foi aconselhada à evicção da colocação do produto sobre a luva e à utilização de medidas protetoras para evitar a exposição aos alergenos. Desde então não teve recidiva da dermatose.

\section{DISCUSSÃO}

Os monómeros acrílicos fazem parte da constituição de diversos materiais usados mais frequentemente pelos dentistas. Os mais frequentes causadores de sensibilização nos dentistas e protésicos dentários são o HEMA e o EGDMA. A elevada prevalência destes alergenos deve-se ao facto destas substâncias existirem na composição dos líquidos de

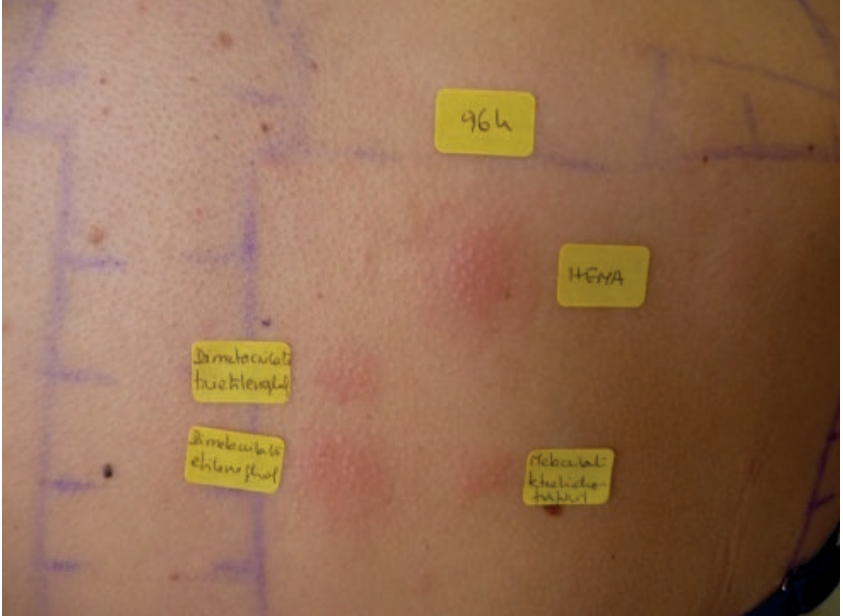

Figura 3 - Provas epicutâneas às 96 h horas com positividades para dimetacrilato de trietilenoglicol (TREGDMA), $2 \%$ vaselina $(++)$; dimetacrilato de etilenoglicol (EGDMA), $2 \%$ vaselina $(++)$; metacrilato de 2 -hidroxietil (HEMA), $2 \%$ vaselina $(+++)$ e metacrilato de tetrahidrofurfuril (THFMA), $2 \%$ vaselina $(++)$.

materiais, sendo os mais comuns os compósitos dentários e as próteses dentárias. ${ }^{3-5}$

A realização de provas epicutâneas pode por em evidência positividade para diferentes metacrilatos. Estas reações resultam de reação cruzada entre os diferentes metacrilatos ou de sensibilização múltipla devido à presença de vários tipos de metacrilatos na composição dos produtos utilizados na restauração dentária. ${ }^{1,3,4,6}$

$\mathrm{Na}$ maioria dos casos relatados de alergia de contacto a metacrilatos nos profissionais dentários as lesões são de eczema das mãos, afetando sobretudo as falanges distais por contacto direto, e de eczema da face, sobretudo das pálpebras quer por contacto com as mãos contaminadas ou por via aerotransportada, dada a volatilidade dos (met)acrilatos.

Neste caso destaca-se a localização atípica da dermite de contacto alérgica causada pela prática habitual individual da doente no manuseamento dos metacrilatos. A doente não relacionou a aplicação do produto com o aparecimento da lesão, pois pressupôs que estava protegida pelo uso de luvas de nitrilo.

Outros casos semelhantes foram descritos em profissionais que tinham por hábito limpar o excesso dos produtos utilizados no dorso da mão. ${ }^{2}$

As luvas mais frequentemente usadas nas práticas profissionais dentárias não oferecem proteção na prevenção da dermite de contacto alérgica. ${ }^{1,2}$ Os monómeros dos metacrilatos têm a capacidade de penetrar as luvas de látex e de vinil em poucos minutos. As luvas de nitrilo oferecem alguma proteção que aumenta com o aumento da espessura da luva. ${ }^{7} \mathrm{O}$ uso de dois pares de luvas de nitrilo ou o uso de luvas de polietileno sob as luvas de nitrilo aumentam a proteção para tarefas que não excedam os 30 a 60 minutos. ${ }^{8}$ As luvas $4 \mathrm{H}^{\circledR}$ (Honeywell Silver Shield) protegem do contacto, mas são pouco maleáveis e inapropriadas para a realização de trabalho minucioso. ${ }^{7}$ 
O uso de instrumentação própria e de medidas protetoras durante a manipulação de produtos com (met)acrilatos é fundamental para a evicção do contacto com as partículas sensibilizantes.

Os (met)acrilatos podem também estar presentes noutros produtos como os geles utilizados na manicure das unhas, em super-colas ou em alguns absorventes dos pensos higiénicos. ${ }^{9}$ Os doentes já sensibilizados devem evitar o contacto com o alergeno na vida quotidiana, evitando, por exemplo, a aplicação de unhas de gel.

\section{CONCLUSÃO}

A localização atípica da manifestação cutânea deste caso demonstra a importância da investigação dos hábitos laborais para a realização do diagnóstico. Os profissionais dentários devem estar informados da necessidade da evicção do contacto com os produtos que contenham metacrilatos a fim de evitar a sensibilização que pode ser limitativa para a sua atividade profissional.

Conflitos de interesse: Os autores declaram não possuir conflitos de interesse. Suporte financeiro: $O$ presente trabalho não foi suportado por nenhum subsídio ou bolsa. Direito à privacidade e consentimento escrito: Os autores declaram que pediram consentimento ao doente para usar as imagens no artigo.

Conflicts of interest: The authors have no conflicts of interest to declare. Financing Support: This work has not received any contribution, grant or scholarship. Privacy policy and informed consent: The authors declare that the patient gave written informed consent for the use of its photos in this article.

\section{REFERÊNCIAS}

1. Ramos L, Cabral R, Gonçalo M. Allergic contact dermatitis caused by acrylates and methacrylates - a 7-year study. Contact Dermatitis. 2014; 71:102-7.

2. Hunasehally RYP, Hughes TM, Stone NM. Atypical pattern of (meth)acrylate allergic contact dermatitis in dental professionals. Br Dent J. 2012; 213:223-4.

3. Teik-Jin Goon A, Bruze M, Zimerson E, Goh CL, Isaksson $M$. Contact allergy to acrylates/methacrylates in the acrylate and nail acrylics series in southern Sweden: Simultaneous positive patch test reaction patterns and possible screening allergens. Contact Dermatitis. 2007; 57:21-7.

4. Aalto-Korte K Alanko K KOJR, Aalto-Korte K, Alanko K, Kuuliala O, Jolanki R. Methacrylate and acrylate allergy in dental personnel. Contact Dermatitis. 2007; 57:324-30.

5. Geukens S, Goossens A. Occupational contact allergy to (meth)acrylates. Contact Dermatitis. 2001; 44:153-9.

6. Sasseville D. Acrylates in contact dermatitis. Dermatitis. 2012; 23:6-16.

7. Andersson $T$, Bruze $M$, Bjorkner B. In vivo testing of the protection of gloves against acrylates in dentin-bonding systems on patients with known contact allergy to acrylates. Contact Dermatitis. 1999; 41:254-9.

8. Sasseville D. Acrylates. Dermatitis. 2012; 23:3-5.

9. Sauder $M$, Pratt $M$. Acrylate systemic contact dermatitis. Dermatitis. 2015; 26:235-8. 